\title{
Structural fluctuation of proteins revealed by terahertz time-domain spectroscopy
}

\author{
Ohki Kambara* and Keisuke Tominaga \\ Molecular Photoscience Research Center, Kobe University, Nada, Kobe, Japan
}

\begin{abstract}
We have measured spectra of the absorption coefficient and refractive index of hen egg white lysozyme in the wavenumber region from $7 \mathrm{~cm}^{-1}$ to $50 \mathrm{~cm}^{-1}$ by terahertz (THz) time-domain spectroscopy. From comparison with the results of the inelastic neutron scattering experiment it is concluded that analysis of the $\mathrm{THz}$ spectra provides information on the vibrational density of states. We studied temperature dependence of the THz spectra as well as hydration effect on them to discuss structural fluctuation of the protein. We observed dynamical transition at around $210 \mathrm{~K}$ for lysozyme.
\end{abstract}

Keywords: Terahertz time-domain spectroscopy, protein, dynamical transition

\section{Introduction}

Protein is one of the biological macromolecules which play a key role in the physiological activities. It is necessary for proteins to exist in an environment with an appropriate $\mathrm{pH}$ and hydration state at proper temperature in order to express their function. According to the theoretical studies, the collective modes of proteins which are relevant to their biological functions have characteristic spectral component in the frequency region below $100 \mathrm{~cm}^{-1}$. Terahertz time-domain spectroscopy (THz-TDS) is one of the ideal methods to investigate dynamics and interactions in this region.

We estimated reduced absorption cross section (RACS) from the absorption cross section $\sigma(\tilde{\nu})$ and the refractive index $n(\tilde{\nu})$, which is expressed as [1]

$$
\begin{aligned}
\sigma_{R}(\tilde{\nu}) & \equiv \frac{\beta h c \tilde{\nu}}{\left(1-\mathrm{e}^{-\beta h c \tilde{\nu}}\right)} n(\tilde{\nu}) \sigma(\tilde{\nu}) \\
& =\frac{2 \pi^{2} c N_{A}}{3 \varepsilon_{0} N} \beta \tilde{\nu}^{2} \int_{-\infty}^{\infty} \mathrm{d} t \mathrm{e}^{-\mathrm{i} 2 \pi c \tilde{\nu} t}\langle\mathbf{M}(0) \cdot \mathbf{M}(t)\rangle,
\end{aligned}
$$

where $\tilde{\nu}$ is the wavenumber, $N$ is the number of molecules in the system, and the other symbols have usual meanings. $\mathbf{M}(t)$ is the total dipole moment of the system. Figure 1(a) shows the time evolution of the electric field of both the sample and reference. From the amplitude change and the shift of the signal as shown in Fig. 1(a), absorption coefficient and refractive index are obtained, respectively. RACS is considered as a product of IR activity and vibrational density of states (VDOS). If we assume that the IR activity is constant in the low-frequency region, the RACS is considered to be proportional to the

\footnotetext{
${ }^{*}$ Corresponding author: Ohki Kambara, Molecular Photoscience Research Center, Kobe University, Nada, Kobe 657-8501, Japan. Tel./Fax: +81 78803 5684; E-mail: kambara@ dolphin.kobe-u.ac.jp.
} 

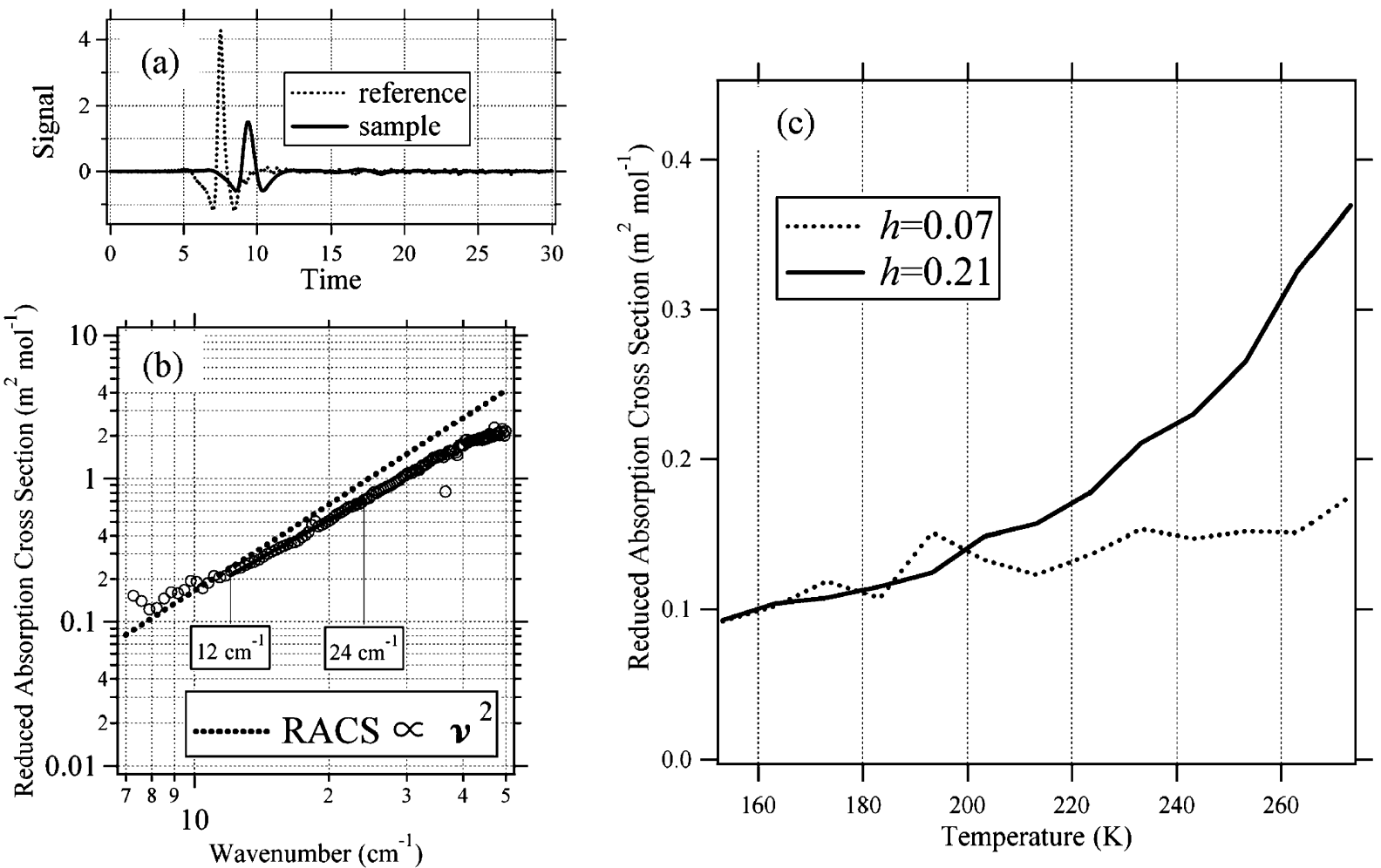

Fig. 1. (a) Waveform of the terahertz electromagnetic wave for the sample (solid line) and reference (dot-line). (b) A double logarithmic plot of the reduced absorption cross section of HEWL as a function of the wavenumber. The dotted line represents a case of $\sigma_{R} \propto \tilde{\nu}^{2}$. (c) Temperature dependence of the reduced absorption cross section of HEWL with different hydration level at $10 \mathrm{~cm}^{-1}$. The hydration level is shown in the figure.

VDOS. In this work, we investigated the low-frequency spectra and structural fluctuation of hen egg white lysozyme (HEWL) by analyzing the RACS.

\section{Materials and methods}

HEWL was purchased from Sigma-Aldrich, Inc. THz spectra were measured by using THz timedomain spectrometer pumped by a mode-locked Ti:sapphire laser. The pulse is centered at $800 \mathrm{~nm}$ with pulse duration of $120 \mathrm{fs}$ and repetition rate of $80 \mathrm{MHz}$. The details of the apparatus were described elsewhere [2]. The optical set up around the sample is covered with a box, and during the measurement dry air is continuously flowed in it.

In order to prepare the powder sample, the solutions of HEWL was prepared first. The concentrations of all the $\mathrm{HEWL}$ solutions were $300 \mu \mathrm{M}$ with a $\mathrm{pH}$ of 7 adjusted by using $\mathrm{HCl}$ and $\mathrm{NaOH}$ solutions. Then, the solutions were frozen by rapid quenching using liquid nitrogen and lyophilized to obtain powder samples. The samples for the measurement were prepared by grinding the powder samples and pressing them to make pellets of $5 \mathrm{~mm}$ diameter. The sample thicknesses are between $500 \mu \mathrm{m}$ and $1000 \mu \mathrm{m}$. To hydrate the pellet samples, they were placed in a box for more than one hour in which humidity is controlled by placing saturated salt aqueous solutions. For the temperature dependence experiment, the 
sample was put in the liquid nitrogen to make it cold. Immediately after taking the sample out from the liquid nitrogen, the sample was placed in the THz-TDS system to start the measurement.

\section{Results and discussion}

\subsection{Power-law behavior of RACS}

HEWL is a protein which is commonly used by spectroscopic studies because it is a small, globular, and soluble protein with high stability. THz spectrum of HEWL without further purification or treatment is measured at ambient temperature $\left(20^{\circ} \mathrm{C}\right)$, and the RACS of HEWL is obtained as shown in Fig. 1(b). The spectrum shows a power-law behavior in the wavenumber region from $7 \mathrm{~cm}^{-1}$ to $50 \mathrm{~cm}^{-1}$. The exponent from $12 \mathrm{~cm}^{-1}$ to $24 \mathrm{~cm}^{-1}$ is estimated to be $1.8\left(\sigma_{R}(\tilde{\nu}) \propto \tilde{\nu}^{1.8}\right)$. The VDOS of HEWL was investigated by incoherent inelastic neutron scattering (INS) in the same spectral region $(1.5-3.0 \mathrm{meV})$ [3]. They reported that the VDOS is proportional to $\tilde{\nu}^{1.8}$, which is consistent with our result. This result directly suggests that the RACS for protein is proportional to the VDOS. In [1] it was reported that the exponents of amino acids were close to two (2.22 \pm 0.03 for L-alanine and $2.12 \pm 0.05$ for glycine), and those for polypeptides were less than two $(1.65 \pm 0.04$ for poly-L-alanine and $1.55 \pm 0.02$ for polyglycine). Since the ideal case of the collection of harmonic oscillators provides an exponent value of two, deviation from two is interpreted as that the low-frequency vibrational modes do not behave harmonically, instead they are coupled with each other by anharmonic interactions. The anharmonic coupling allows protein to change its confirmation largely, which is a key to the expression of the protein function.

\subsection{Dynamical transition}

We investigated temperature dependence of the THz spectra of HEWL with different hydration conditions to examine whether the low-frequency dynamics of the protein is influenced by temperature and/or hydration condition. The temperature dependence of the RACS was studied for $h=0.07$ and 0.21 , where $h$ is the weight of the hydrated water in grams per one gram of the protein. Here we focus on the intensity of the RACS at certain wavenumber. Figure 1(c) summarizes the temperature dependence of the RACS intensities for the HEWL sample prepared at $\mathrm{pH}=7$. For the relatively dried sample ( $h=0.07$ ), the intensities of the RACS gradually increased as a function of temperature. This dependence can be interpreted in terms of Eq. (1) in a qualitative level. The RACS is proportional to the Fourier spectrum of the time-correlation function of $\mathbf{M}(t)$. We here simply assume an exponential function for the TCF, $\langle\mathbf{M}(t) \mathbf{M}(0)\rangle=M^{2} \mathrm{e}^{-t / \tau}$. The pre-exponential factor $M^{2}$ corresponds to the magnitude of the total dipole moment, which is a sum of the amplitudes of the oscillators in the system. Increase of temperature induces thermal activation of the oscillators, which increases the intensity of the RACS. When the hydration level is 0.21 , the temperature dependence of the RACS intensity is similar to the dried one from $160 \mathrm{~K}$ to $210 \mathrm{~K}$. However, above $210 \mathrm{~K}$ the hydrated sample shows larger temperature dependence than the dried one. This suggests that in this temperature range the atomic motions in the protein are more active. Such large-amplitude motions may induce a structural change which is necessary for the expression of the function.

This transition, often called dynamical transition or glass transition, is generally found in proteins, which has been observed by NMR spectroscopy [4], X-ray diffraction experiment [5], and Mossbauer spectroscopy [6]. Recently, there are a few reports on discussion of the dynamical transition measured 
by THz-TDS [7]. The mechanism of the dynamical transition is following. There are multiple quasistable structures of protein in its free-energy surface. Below the transition temperature localized motions trapped in the quasi-stable structures are dominant, whereas above the transition temperature the interconversion between the quasi-stable structures are thermally activated, which consequently leads to the large-amplitude motions in the protein. Some proteins manifest their functions only above the dynamical transition temperature, suggesting that the dynamical transition is strongly related to the protein functions. Furthermore, this transition is only observed for the hydrated samples, and not observed in the dried sample. Therefore, hydration waters around the protein play important role in the dynamical transition.

\section{Conclusions}

We have measured spectra of the absorption coefficient and refractive index of HEWL in the wavenumber region from $7 \mathrm{~cm}^{-1}$ to $50 \mathrm{~cm}^{-1}$ by THz-TDS. The major concern in this study is whether the $\mathrm{THz}$ spectra can provide any information regarding the structural fluctuation relevant to the protein function. We found that analysis of the THz spectra provides information on the VDOS in the lowfrequency region from comparison with the inelastic neutron scattering experiment. From the experiment of the temperature dependence and hydration effects we observed dynamical transition at around $210 \mathrm{~K}$ for the relatively hydrated sample of HEWL.

\section{Acknowledgement}

This work was supported by a Grant-in-Aid for Creative Scientific Research (No. 17GS0209) from the Ministry of Education, Culture, Sports, Science, and Technology of Japan.

\section{References}

[1] K. Yamamoto, K. Tominaga, H. Sasakawa, A. Tamura, H. Murakami, H. Ohtake and N. Sarukura, Terahertz time-domain spectroscopy of amino acids and polypeptides, Biophys. J. 89 (2005), L22-L24.

[2] P. Dutta and K. Tominaga, Obtaining low frequency spectra of acetone dissolved in cyclohexane by terahertz time-domain spectroscopy, J. Phys. Chem. A 113 (2009), 8235-8242.

[3] S.G. Lushnikov, A.V. Svanidze and I.L. Sashin, Vibrational density of states of hen egg white lysozyme, JETP Lett. 82 (2005), 30-33.

[4] M. Lagi, X. Chu, C. Kim, F. Mallamace, P. Baglioni and S.-H. Chen, The low-temperature dynamic crossover phenomenon in protein hydration water: Simulation vs. experiments, J. Phys. Chem. B 112 (2008), 1571-1575.

[5] D. Ringe and G.A. Petsko, The 'glass transition' in protein dynamics: what it is, why it occurs, and how to exploit it, Biophys. Chem. 105 (2003), 667-680.

[6] H. Lichtenegger, W. Doster, T. Kleinert, A. Birk, B. Sepiol and G. Vogl, Heme-solvent coupling: A Mossbauer study of myoglobin in sucrose, Biophys. J. 76 (1999), 414-422.

[7] Y. He, P.I. Ku, J.R. Knab, J.Y. Chen and A.G. Markelz, Protein dynamical transition does not require protein structure, Phys. Rev. Lett. 101 (2008), 178103-1-4. 


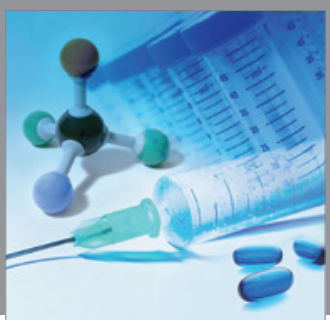

International Journal of

Medicinal Chemistry

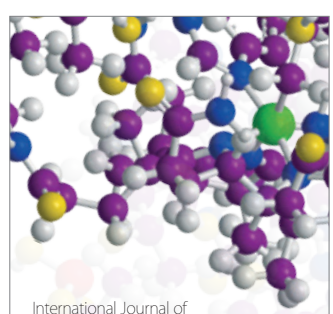

Carbohydrate Chemistry

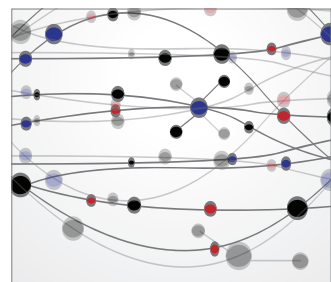

The Scientific World Journal
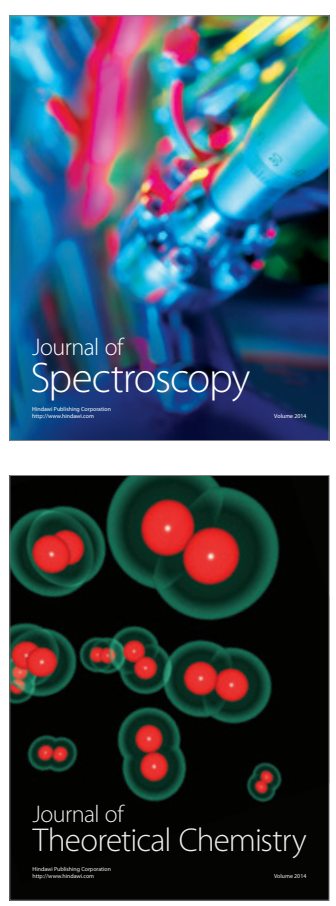
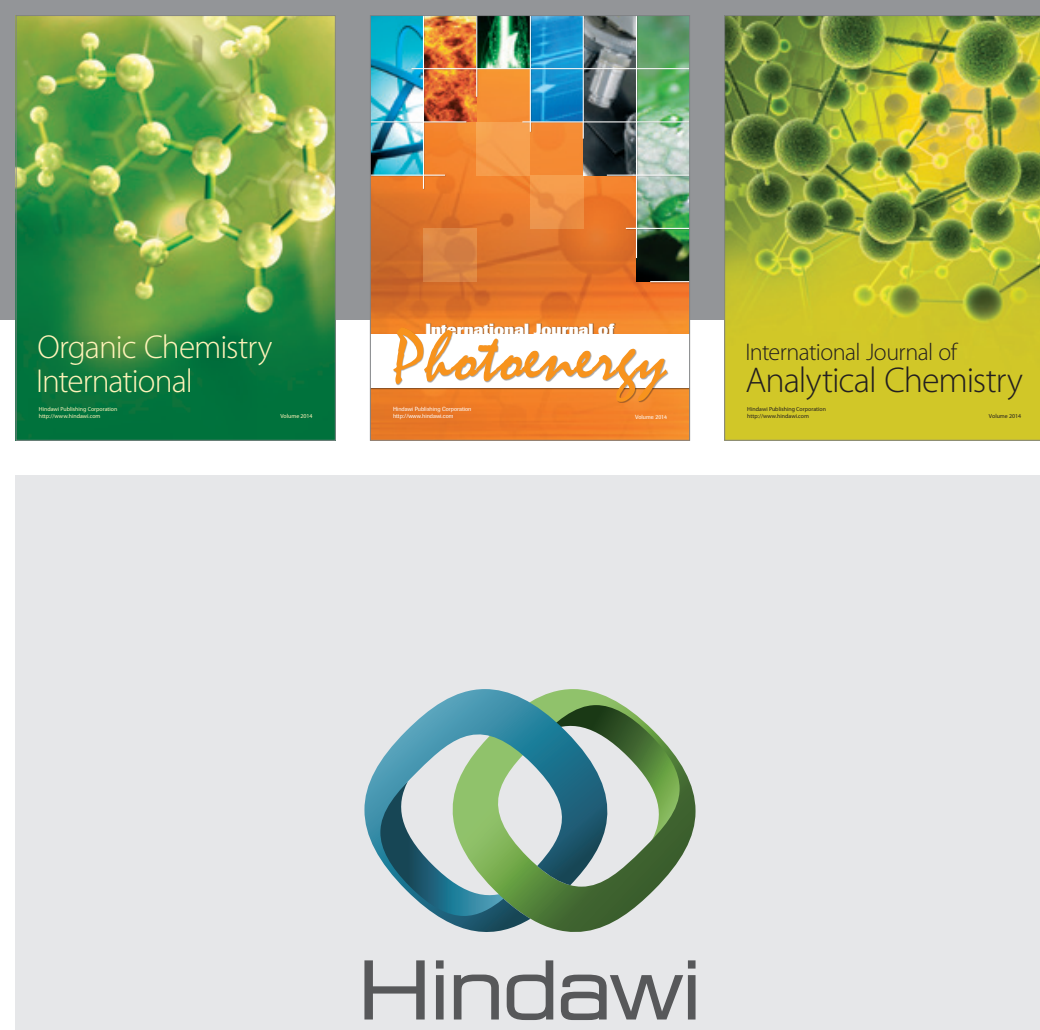

Submit your manuscripts at

http://www.hindawi.com
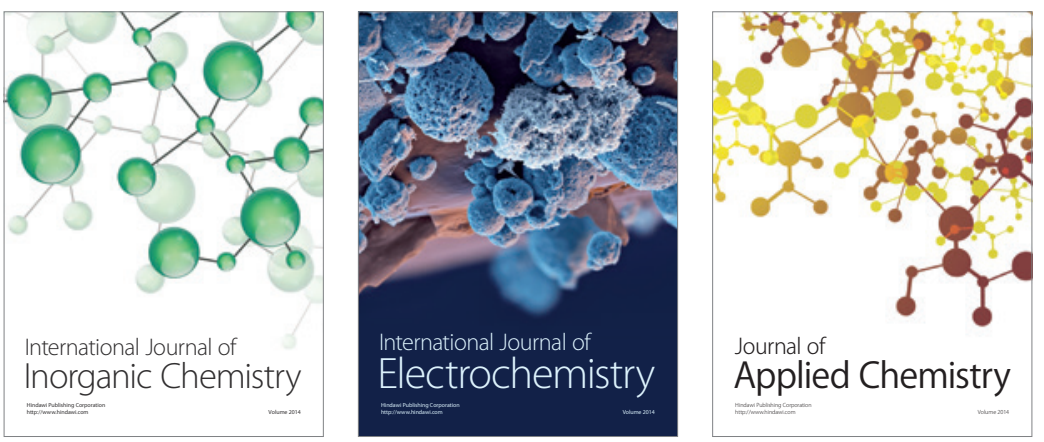

Journal of

Applied Chemistry
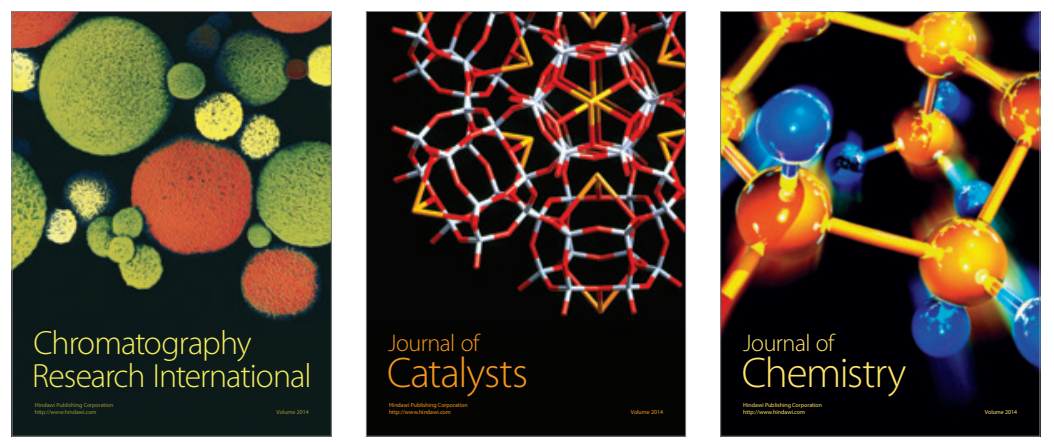
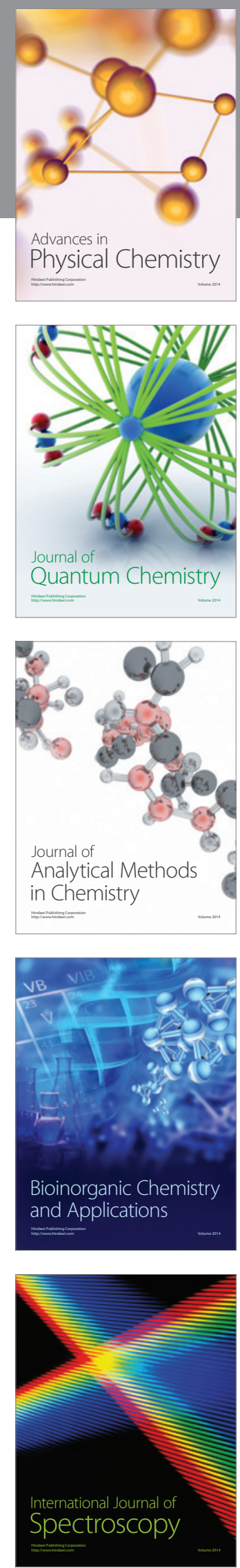